
\title{
25 Research Soure \\ The Change in Retinal Ellipsoid Zone Thickness in Patients with Reticular Macular Disease
}

\section{Yuyao Diao}

Guangzhou Medical College First Affiliated Hospital

Hua Hao

University of Southern California Keck School of Medicine

Lu Cheng

Zhongshan University Affiliated Eye Hospital: Sun Yat-Sen University Zhongshan Ophthalmic Center

\section{Varun Pattisapu}

Cedars-Sinai Heart Institute

Hao Huang

Guangzhou Medical College First Affiliated Hospital

\section{Yongzhao Wei}

Guangzhou Medical College First Affiliated Hospital

Hao Cheng ( $\nabla$ chrischenghao@gzhmu.edu.cn )

The First Affiliated Hospital of Guangzhou Medical University https://orcid.org/0000-0003-4716-1963

\section{Research Article}

Keywords: Reticular macular disease, Reticular pseudodrusen, Ellipsoid zone, SD-OCT, Age-related macular disease

Posted Date: April 26th, 2021

DOI: https://doi.org/10.21203/rs.3.rs-371564/v1

License: () (1) This work is licensed under a Creative Commons Attribution 4.0 International License. Read Full License 


\section{Abstract}

Purpose: Compare the thickness of the retinal ellipsoidal zone in patients with Reticular Macular Disease (RMD) to a control population.

Methods: This case-control study was conducted on patients diagnosed with RMD at the First Affiliated Hospital of Guangzhou Medical University from April 2018 to November 2018. We identified 25 cases who were diagnosed with RMD and 20 normal control eyes. Divide the posterior pole into five regions (superior, inferior, nasal, temporal, and subfoveal zones), and manually measure the five regions posterior ellipsoidal zone thickness, all the images were obtained by SD-OCT. Generalized estimating equation models were used to control for potential confounders and within-subject variation.

Results: The mean ellipsoidal zone thickness in RMD patients five regions were all significantly lower than in the control group (all $\mathrm{P}<0.01$ ). The mean ellipsoidal zone thickness value in men with RMD has a more significant change than females. Furthermore, the mean ellipsoidal zone thickness of reticular pseudodrusen (RPD) eyes with drusen were not significantly different from the pure RPD eyes $(P=0.34)$.

Conclusions: Our findings reveal that the mechanism of RMD occurs may be related to damage to the mitochondria of photoreceptor cells, which may be one of the mechanisms that RMD occurs. When the impairment degree of mitochondria reach to a certain level, may lead to further vision loss in RMD patients. Therefore, to protect the retinal ellipsoidal zone and its mitochdrial early is important to prevent further impairment of visual function and vision loss.

\section{Background}

Reticular macular disease (RMD) is a new type of reticular disease, with the characteristic of reticular pseudodrusen (RPD), also known as subretinal drusen deposit (SDD)(1). RPD was first discovered by Mimoun et al. in 1990 by blue light fundus photography, which showed a yellow network change in the macular area(2). In spectral-domain optical coherence tomography (SD-OCT), RPD is characterized by high-reflection sediments starting from the retinal pigment epithelium (RPE) (3). SD-OCT, infrared photographs (IR), and multi-color imaging $(\mathrm{MCl}$ ) have high sensitivity and specificity for RPD(4). RPD and the better-known drusen are considered to be closely related to the massive loss of retinal pigment epithelium and photoreceptor damage, but the initiation of drusen is between the Bruch membrane and the choroid, and RPD starts above the RPE(5).

RMD is associated with a variety of fundus diseases, particularly age-related macular disease (AMD), the leading cause of blindness in the elderly, and is considered to be an essential factor in promoting advanced AMD(6). However, the pathogenesis of RMD is still in the exploratory stage. Studies have shown that RPD eyes have significant defects in dark adaptation, and also have a significantly greater mean rod-intercept time (RIT) than in AMD eyes without RPD (7). The destruction of the photoreceptor inner segment/outer segment (IS/OS) layer, the ellipsoid zone (EZ), may cause poor visual function in the patient(8), and the destruction of the EZ is including the destruction of its continuity and the thickness of it becoming thinner. Exploring the association of RMD with changes in the thickness of the EZ will improve the understanding of RMD pathogenesis and its clinical manifestations. 


\section{Methods}

\section{Study Population}

The general study design was retrospective and case-control. All cases included in this study had RPD (the RMD diagnostic necessary condition), and nine eyes also with drusen in the case group. RMD diagnostic criteria are 1. It was found on SD-OCT that high-reflection deposits, RPD, occurred between the RPE and the chimeric band. 2 . In the IR, the lesion appears as a low-reflective lesion or a highly reflective lesion on the surrounding mildly highreflective background, and the lesion can be connected into a network(9). 3. RPD manifests as yellow-green lesions in $\mathrm{MCl}(1)$. Patients with evidence of age-related macular degeneration, retinal detachment, diabetic retinopathy, vitreous hemorrhage, high myopia and pathological myopia retinal changes, severe cataract failure A clear view of the fundus, retinal arteriovenous occlusion, macular edema, endophthalmitis, history of ocular trauma, glaucoma patients and patients with a history of fundus surgery were excluded. The case was identified from the First Affiliated Hospital of Guangzhou Medical University and was evaluated between April 2018 to November 2018. The visual acuity testing, intraocular pressure measurement, refraction measurement, slit-lamp microscopy, SD-OCT, infrared photography, $\mathrm{MCl}$, and Fundus photography were reviewed to confirm the diagnosis. Cases that did not conform to the case definitions or for whom complete ophthalmic history (including medications, medical history, and social history) and examinations were not available were excluded.

A control group of patients matched for gender and age with ophthalmic conditions other than RMD was assembled. All controls had complete ocular history and examinations performed, and was selected in the same period with the case group. Patients with retinal disorders other than RMD were included as controls.

\section{Examination and Imaging}

All participants underwent a complete ophthalmoscopic examination, including visual acuity with the ETDRS chart, measurement of intraocular pressure, slit-lamp examination. The fundus examination was after the pupils were fully dispersed with Tropicamide Phenylephrine Eye Drops. Color fundus photos were acquired with the TRC-50DX retinal camera (Topcon Medical Systems, Tokyo, Japan). Infrared reflectance (scan angle $30^{\circ}$; image resolution, $768^{*} 768$ pixels $\llbracket$ and $\mathrm{MCl}$ and spectral-domain optical coherence tomography (scan angle, $30^{\circ}$; scan depth, $1.9 \mathrm{~mm}$; scan length, $8.9 \mathrm{~mm}$; digital depth resolution, approximately $3.87 \mu \mathrm{m}$ per pixel image resolution; ART mode, 100 images averaged) scans were acquired with the Heidelberg Spectralis (Heidelberg Engineering, Heidelberg, Germany).

Both the horizontal $\left(0^{\circ}\right)$ and vertical $\left(90^{\circ}\right)$ sections passing directly through the center of the fovea were used for the ellipsoid zone thickness measurements. Ellipsoid zone is a hyperreflective line above the retinal pigment epithelium. We defined the thickness of the ellipsoid zone was the distance between the upper edge and the lower edge of the hyperreflective line. Ellipsoid zone thickness measurements were obtained manually using the scale supplied with the software (Adobe Photoshop CC, 19.1.6) at $13 \mathrm{~mm}$ intervals from the fovea to a distance of $65 \mathrm{~mm}$ in the nasal, temporal, superior, and inferior directions (the setting of the equidistance line is set by the software). (Figure 1). The average value of the 22 thicknesses measured at each point is statistically analyzed as the Ellipsoid zone thickness(10). SD-OCT examination and thickness measurement of all patients were performed by one person.

\section{Statistics Analysis}


Statistical analysis was performed with SAS 9.4 (SAS Institute, Cary, NC, USA). The generalized estimating equation model was used to compare the thickness of the EZ in RMD group to the normal control group and mean differences of the thickness of the EZ between male and female, and the EZ of the central fovea thickness differences for RMD group and the normal control group. The Wilcoxon rank-sum test was used to calculate the RMD eyes group and the (RMD +drusen) eye group. A P-value of less than 0.05 was considered as statistically significant.

\section{Results}

\section{Baseline Characteristics}

RMD group contained 43 eyes of 25 patients ( 9 eyes with drusen), and the normal control group contained 40 eyes of 20 patients. The mean age was $68.20 \pm 12.37$ years in the RMD group and $41.21 \pm 11.32$ years in the normal control group (Table 1).

Table 1. Basic characteristics of Reticular Macular Disease (RMD) group and normal control group.

\begin{tabular}{|lllll|}
\hline Groups & eyes & Age (year) & \multicolumn{2}{c|}{ Gender (patients) } \\
\cline { 4 - 5 } & & & male & female \\
\hline RMD group & 43 & $68.2 \pm 12.37$ & 14 & 11 \\
\hline Control group & 40 & $62.8 \pm 10.24$ & 8 & 12 \\
\hline
\end{tabular}

\section{Ellipsoid Zone Thickness}

The thickness of the RMD group (mean: $13.53 \mu \mathrm{m}$ ) was significantly thinner than the normal control group (mean: 17.95 $\mu \mathrm{m})$ after adjusted age and gender in our model $(P<0.001)$ (Figure 2A).

Table 2. Ellipsoidal Zone Thickness in Different Quadrants of Reticular Macular Disease (RMD) Patients.

\begin{tabular}{|c|c|c|c|c|c|c|c|}
\hline \multirow[t]{2}{*}{ Group } & \multirow{2}{*}{$\begin{array}{l}\text { Number } \\
\text { of Eyes }\end{array}$} & \multirow[t]{2}{*}{ Age (year) } & \multicolumn{5}{|c|}{ Ellipsoid Zone Thickness (mm) } \\
\hline & & & Superior & Inferior & Nasal & Temporal & Subfoveal \\
\hline $\begin{array}{l}\text { RMD } \\
\text { group }\end{array}$ & 43 & $68.2 \pm 12.37$ & $12.87 \pm 6.35$ & $13.02 \pm 6.63$ & $14.03 \pm 6.45$ & $14.18 \pm 6.53$ & $16.12 \pm 7.62$ \\
\hline $\begin{array}{l}\text { Control } \\
\text { group }\end{array}$ & 40 & $62.8 \pm 10.24$ & $18.04 \pm 3.27$ & $17.16 \pm 3.61$ & $18.50 \pm 3.15$ & $18.09 \pm 2.98$ & $22.22 \pm 3.44$ \\
\hline P-value & & & $<0.01$ & $<0.01$ & $<0.01$ & $<0.01$ & $<0.01$ \\
\hline $\begin{array}{l}\text { Model b- } \\
\text { Estimate }\end{array}$ & & & 4.65 & 3.78 & 4.14 & 3.38 & 5.61 \\
\hline
\end{tabular}

The thickness of the subfoveal EZ in the RMD group (mean: $16.12 \mu \mathrm{m}$ ) was significantly thinner than the normal control group (mean: $22.22 \mu \mathrm{m}$ ) after adjusted age and gender in our model $(P<0.001)$ (Figure 3 and Table 2). 
Different quadrants EZ thickness of the control group and of the RMD group were compared. The mean EZ thickness values in superior, inferior, nasal, and temporal were $18.04 \pm 3.27 \mu \mathrm{m}, 17.16 \pm 3.61 \mu \mathrm{m}, 18.50 \pm 3.15 \mu \mathrm{m}$, $18.09 \pm 2.98 \mu \mathrm{m}$, and in the control group were $12.87 \pm 6.35 \mu \mathrm{m}, 13.02 \pm 6.63 \mu \mathrm{m}, 14.03 \pm 6.45 \mu \mathrm{m}, 14.18 \pm 6.53 \mu \mathrm{m}$. The mean EZ thickness values in superior, inferior, nasal, and temporal of the RMD group were significantly thinner than in the control group (all at $\mathrm{P}<0.01$ ). The difference between the two groups in a superior quadrant was the most significant among the four quadrants. All the results were adjusted age and gender in our model (Figure 3 and Table 2).

\section{Ellipsoid Zone Thickness by Gender}

The average thickness of the posterior ellipsoidal zone of male RMD patients was compared with that of the male in the healthy control group. The average thickness of the EZ of male RMD patients was $12.22 \pm 7.38 \mu \mathrm{m}$, and that of males in the control group was $17.84 \pm 3.37 \mu \mathrm{m}$. Compared with normal males, men with RMD had significantly thinner EZ thickness, $\mathrm{P}<0.01$. Comparing the average thickness of the $\mathrm{EZ}$ of female RMD patients with the female in the healthy control group, the average thickness of EZ of female RMD patients was $15.19 \pm 4.69 \mu \mathrm{m}$, and that of the control group was $18.02 \pm 3.24 \mu \mathrm{m}$. The average degree of the EZ was thinner in women with RMD compared with healthy women; the result is marginally significant, $P=0.07$. The effect of RMD on male EZ thickness is greater than that of the female (Figure 2B).

\section{Influence of Drusen to Ellipsoid Zone Thickness}

In order to detect whether the thickness of the RMD eye EZ with the drusen is different from the thickness of the ellipsoid zone of the pure RMD eye, thereby producing the effect of the drusen on the experimental results, the rank-sum test is used for the pure RMD eye (The difference in the average EZ thickness of 34 eyes) and RMD eyes with drusen ( 9 eyes) was analyzed. After controlling the effect of age and gender on the results, $P=0.34$. There was no significant difference in the thickness of the EZ between the RMD eye with the drusen and the pure RMD eye.

\section{Discussion}

RMD is thought to be closely related to AMD. The mechanism of RMD is still under investigation. In this study, comparing between 43 RMD eyes EZ thickness and 40 healthy eyes EZ thickness. Our results demonstrate that the EZ thickness of RMD patients is thinner than in healthy subjects. Many studies suggest that RMD is closely related to choroidal lesions $(11,12)$. Cheng $\mathrm{H}$ et al. found that patients younger than 82 years with AMD and RPD had a significantly smaller mean choroidal thickness than in AMD alone (MD-53.72 $\mu \mathrm{m}, \mathrm{P}=0.01)(12)$.

Chatziralli I et al. found that the degree of chorionic capillary morphological damage in patients with RMD was higher than that of patients with the same drusen size, and choroidal capillary changes may occur in patients with RMD(11). Because choroidal circulation is the source of blood and oxygen supply to the outer retina, it is essential for maintaining various functions of extra-retinal cells. Therefore, according to the existing research, it can be inferred that RMD may be closely related to choroidal blood circulation, which is consistent with various pathophysiological mechanisms caused by the decrease of posterior blood flow and choroidal structure(13).

Besides choroid-related lesions, studies have shown that RPD is closely related to the damage of the Bruch membrane, and there is a close relationship between pseudoxanthoma elasticum and adult-onset foveomacular vitelliform dystrophy(AFVD) and Sorsby fundus dystrophy. RPD may be caused directly or indirectly damage by 
Bruch membrane damage $(9,14,15)$. Some researchers have found that patients with RPD have significantly reduced macular visual sensitivity compared with patients with typical drusen(16, 17), Alten $\mathrm{F}$ et al. used multifocal electroretinography (mf-ERG) and SD-OCT to study the structure of RPD eyes, and functional correlation found that RPD eye visual function decreased over time(18). This means that the cause of RPD is not monofactorial, and the occurrence of RMD is not only accompanied by changes in the choroid but also by the changes in the EZ. The EZ is full of cones and rods, and the high reflection of this band is mitochondria. These two kinds of cells have the characteristic that the disc of them will continue to fall off and synthesize, which tightly related to the mitochondria(19). Therefore, our study measures EZ thickness of the main visual function layer of the retina to explore whether RPD eyes have changes in EZ thickness. The choroidal affecting theory is approbated widely. Our research opens up a new way to explore RMD pathogenesis.

The ellipsoidal zone is what is known as the IS/OS layer. This band contains the inner section of the photoreceptor and is the main visual function layer on the retina(20). The degree of damage to the EZ in most research is based on its integrity and the intensity of the reflection on the OCT. It has been reported that AMD eyes with RPD have ellipsoidal lesions, which is related to cone density reduction(21). Spaide et al. found that eye progression was associated with geographic atrophy and the damage of photoreceptor cell length and destruction of the EZ or the intensity decrease of the EZ(22). However, the measurement of the thickness of the EZ has not been reported.

In the present study, RMD patients have a thinner EZ thickness compared with the normal group $(P<0.01)$. The $E Z$ is the main area where the photoreceptors are located. The photoreceptors are mainly composed of cones and rods. RMD patients' EZ becoming thinner means that the photoreceptors have changed in structure. We postulate this is due to the reduction or atrophy of the cones in this layer. The choroid of RMD patients has obvious capillary lesions, which can directly affect the blood supply of the outer retina(11). Corvi F believes that the occurrence of RPD is related to the shrinkage or fibrosis of choroidal vessels(23). Perhaps it is because the lesion of the choroidal vascular layer leads to insufficient blood supply to the visual function layer, resulting in a decrease or shrinkage of the number of visual function cells.

Furthermore, the current study found that EZ in the central fovea of the RMD patients was significantly thinner than in the control group $(P<0.01)$. In the central fovea of the macula, has central cone cell bundle which is the most accurate and sensitive part of the macular vision. The damage of cone cells may lead to vision loss, visual distortion, color vision abnormalities. The EZ becomes thinner in the central fovea of the RMD patient, which indicates that the cone cell density is decreased in this region. Wu Z et al. measured the relative intensity of the EZ to study the changes in the inner segment of early $\operatorname{AMD}(24)$. The results showed that the EZ reflection intensity of the AMD was significantly lower than in the control group. The thinner EZ and the decreasing intensity of the EZ reflection are all the manifestations of the structure and quantity reduction of visual functional cells. RMD is an essential factor in progression from early to late stage AMD(6). Our experiment results confirm that both RMD and early AMD have ellipsoidal changes, and they may have similar pathogenesis. It also confirms that RMD affects the visual function layer or that RMD is not only accompanied by choroidal changes, but also structural changes in the visual function layer.

In this study, we measured and compared the EZ thickness in the superior, inferior, nasal, and temporal quadrants between the RMD group and the control group and found that the RMD group's EZ is significantly thinner in all quadrants with the superior quadrant in particular having the thinnest zone. RMD mostly occurs 
between the superior and upper temporal artery ${ }^{6}$. Thus, according to our result, the most affected location of the EZ thickness coincides with the predilection site of RMD, which may because that the choroidal blood vessels in superior are more susceptible to damage. However, compared with the change of superior with RMD, the change in the central fovea was greater. Since the central fovea contains only cones and the cone density decreases from the center to peripheral retina, it can be deduced that RMD may decrease the number of cones and lead to atrophy of cones, and RMD patients change on visual function cells mainly acts on cones. However, the specific mechanism still needs further study.

In addition, the study finds that the EZ of RMD males was significantly thinner than in the healthy males $(P<0.01)$; female RMD patients' EZ was thinner than the healthy males with a trend towards significance $(P=0.07)$. According to this result, we may conclude that RMD affects men more than women. The PRD pathology was analyzed by Rudolf $\mathrm{M}$ et al. found that RPD lesions contained a large quantity of unesterified cholesterol(25). HDL can reduce free cholesterol in the blood and reduce the deposition of cholesterol in the tissue. Therefore, the effect of RMD on men is greater than that of women.

In the RMD group of this study, nine eyes were accompanied by drusen. Comparing pure RMD eyes with the drusen RMD eyes, the EZ thickness of the pure RMD eyes was not significantly different from the drusen RMD eyes $(P=0.34)$. Curcio CA pointed out that the occurrence of drusen and RPD is closely related to the material exchange pathway between cones, rods, RPE, Müller cells, and choroidal capillary endothelium, so drusen may have correlation with the change of EZ thickness(5). Our results show that RMD eyes with or without drusen have no difference in EZ thickness.

There are several limitations to our study. First, the EZ thickness was measured manually, which can lead to some deviations so that we cannot obtain the accurate value about the EZ thickness. Second, the sample size is small and without the follow-up to determine whether the thickness will change in a different stage of RMD and when the EZ thickness changed. Though we compared the EZ thickness between the RMD patients with and without RPD, the sample size between these two groups is a great disparity. Third, several types of research have reported that RMD may accompany some systemic disease, but we did not consider whether this systemic factor can influence our results.

In conclusion, The EZ presents hyperreflections on SD-OCT is lead by photoreceptors' mitochondria of photoreceptor cells, which are the key parts of the retinal structure converting light energy into electrical impulses, and is relative to visual function. This finding demonstrates a novel method of studying the pathogenesis of RMD. Our findings reveal that the mechanism of RMD occurs may be related to damage to the mitochondria of photoreceptor cells, which may be one of the mechanisms that RMD occurs. When the impairment degree of mitochondria reach to a certain level, may lead to further vision loss in RMD patients. Therefore, to protect the retinal ellipsoidal zone and its mitochdrial early is important to prevent further impairment of visual function and vision loss. However, the molecular mechanism is not to be confirmed. Further laboratory research will be required. Also, the sample size needs to be expanded, and the measurement method will be optimized to make further exploration and research.

\section{Declarations}

\section{Ethics approval and consent to participate}


The study was approved by the Institutional Review Board of The First Affiliated Hospital of Guangzhou Medical University.

\section{Consent for publication}

Not applicable

\section{Availability of data and materials}

The datasets generated and analysed during the current study are not publicly available due reason why data are not public but are available from the corresponding author on reasonable request.

\section{Competing interests}

The authors declare that they have no competing interests.

\section{Funding}

This work was funded by the Special Fund for Public Welfare Research and Capacity Construction in Guangdong, China, under grant number 2014A020212365.

\section{Authors' contributions}

DYY designed the research and collect the patient data, and was a major contributor in writing the manuscript. $\mathrm{HH}$ interpreted the patient data and attended manuscript writing. CL and WYZ collected the patient data, VP attended manuscript writing, $\mathrm{CH}$ provided all the technic guide. All authors read and approved the final manuscript.

\section{Acknowledgements}

Not applicable

\section{References}

1. Suzuki M, Sato T, Spaide RF. Pseudodrusen subtypes as delineated by multimodal imaging of the fundus. Am J Ophthalmol. 2014;157(5):1005-12.

2. Mimoun G, Soubrane G, Coscas G. [Macular drusen]. J Fr Ophtalmol. 1990;13(10):511-30.

3. Zweifel SA, Spaide RF, Curcio CA, Malek G, Imamura Y. Reticular pseudodrusen are subretinal drusenoid deposits. Ophthalmology. 2010;117(2):303-12.e1.

4. De Bats F, Mathis T, Mauget-Faÿsse M, Joubert F, Denis P, Kodjikian L. PREVALENCE OF RETICULAR PSEUDODRUSEN IN AGE-RELATED MACULAR DEGENERATION USING MULTIMODAL IMAGING. Retina. 2016;36(1):46-52.

5. Curcio CA. Soft Drusen in Age-Related Macular Degeneration: Biology and Targeting Via the Oil Spill Strategies. Invest Ophthalmol Vis Sci. 2018;59(4):Amd160-amd81.

6. Smith RT, Sohrab MA, Busuioc M, Barile G. Reticular macular disease. Am J Ophthalmol. 2009;148(5):73343.e2. 
7. Flamendorf J, Agrón E, Wong WT, Thompson D, Wiley HE, Doss EL, et al. Impairments in Dark Adaptation Are Associated with Age-Related Macular Degeneration Severity and Reticular Pseudodrusen. Ophthalmology. 2015;122(10):2053-62.

8. Oster SF, Mojana F, Brar M, Yuson RM, Cheng L, Freeman WR. Disruption of the photoreceptor inner segment/outer segment layer on spectral domain-optical coherence tomography is a predictor of poor visual acuity in patients with epiretinal membranes. Retina. 2010;30(5):713-8.

9. Gliem M, Hendig D, Finger RP, Holz FG, Charbel Issa P. Reticular pseudodrusen associated with a diseased bruch membrane in pseudoxanthoma elasticum. JAMA Ophthalmol. 2015;133(5):581-8.

10. Wang NK, Lai CC, Chu HY, Chen YP, Chen KJ, Wu WC, et al. Classification of early dry-type myopic maculopathy with macular choroidal thickness. Am J Ophthalmol. 2012;153(4):669-77, 77.e1-2.

11. Chatziralli I, Theodossiadis G, Panagiotidis D, Pousoulidi P, Theodossiadis P. Choriocapillaris' alterations in the presence of reticular pseudodrusen compared to drusen: study based on OCTA findings. Int Ophthalmol. 2018;38(5):1887-93.

12. Cheng H, Kaszubski PA, Hao H, Saade C, Cunningham C, Freund KB, et al. The Relationship Between Reticular Macular Disease and Choroidal Thickness. Curr Eye Res. 2016;41(11):1492-7.

13. Alten F, Clemens CR, Heiduschka P, Eter N. Localized reticular pseudodrusen and their topographic relation to choroidal watershed zones and changes in choroidal volumes. Invest Ophthalmol Vis Sci. 2013;54(5):32507.

14. Gliem M, Müller PL, Mangold E, Bolz HJ, Stöhr H, Weber BH, et al. Reticular Pseudodrusen in Sorsby Fundus Dystrophy. Ophthalmology. 2015;122(8):1555-62.

15. Wilde C, Lakshmanan A, Patel M, Morales MU, Dhar-Munshi S, Amoaku WM. Prevalence of reticular pseudodrusen in newly presenting adult onset foveomacular vitelliform dystrophy. Eye (Lond). 2016;30(6):817-24.

16. Querques G, Querques L, Forte R, Massamba N, Coscas F, Souied EH. Choroidal changes associated with reticular pseudodrusen. Invest Ophthalmol Vis Sci. 2012;53(3):1258-63.

17. Sivaprasad S, Bird A, Nitiahpapand R, Nicholson L, Hykin P, Chatziralli I. Perspectives on reticular pseudodrusen in age-related macular degeneration. Surv Ophthalmol. 2016;61(5):521-37.

18. Alten F, Heiduschka P, Clemens CR, Eter N. Longitudinal structure/function analysis in reticular pseudodrusen. Invest Ophthalmol Vis Sci. 2014;55(9):6073-81.

19. Coco RM, Tellería JJ, Sanabria MR, Rodríguez-Rúa E, García MT. PRPH2 (Peripherin/RDS) mutations associated with different macular dystrophies in a Spanish population: a new mutation. Eur J Ophthalmol. 2010;20(4):724-32.

20. Deye N, Vincent F, Michel P, Ehrmann S, da Silva D, Piagnerelli M, et al. Changes in cardiac arrest patients' temperature management after the 2013 "TTM" trial: results from an international survey. Ann Intensive Care. 2016;6(1):4.

21. Mrejen S, Sato T, Curcio CA, Spaide RF. Assessing the cone photoreceptor mosaic in eyes with pseudodrusen and soft Drusen in vivo using adaptive optics imaging. Ophthalmology. 2014;121(2):545-51.

22. Spaide RF. Outer retinal atrophy after regression of subretinal drusenoid deposits as a newly recognized form of late age-related macular degeneration. Retina. 2013;33(9):1800-8. 
23. Corvi F, Souied EH, Capuano V, Costanzo E, Benatti L, Querques L, et al. Choroidal structure in eyes with drusen and reticular pseudodrusen determined by binarisation of optical coherence tomographic images. $\mathrm{Br}$ J Ophthalmol. 2017;101(3):348-52.

24. Wu Z, Ayton LN, Guymer RH, Luu CD. Relationship between the second reflective band on optical coherence tomography and multifocal electroretinography in age-related macular degeneration. Invest Ophthalmol Vis Sci. 2013;54(4):2800-6.

25. Rudolf M, Malek G, Messinger JD, Clark ME, Wang L, Curcio CA. Sub-retinal drusenoid deposits in human retina: organization and composition. Exp Eye Res. 2008;87(5):402-8.

\section{Figures}

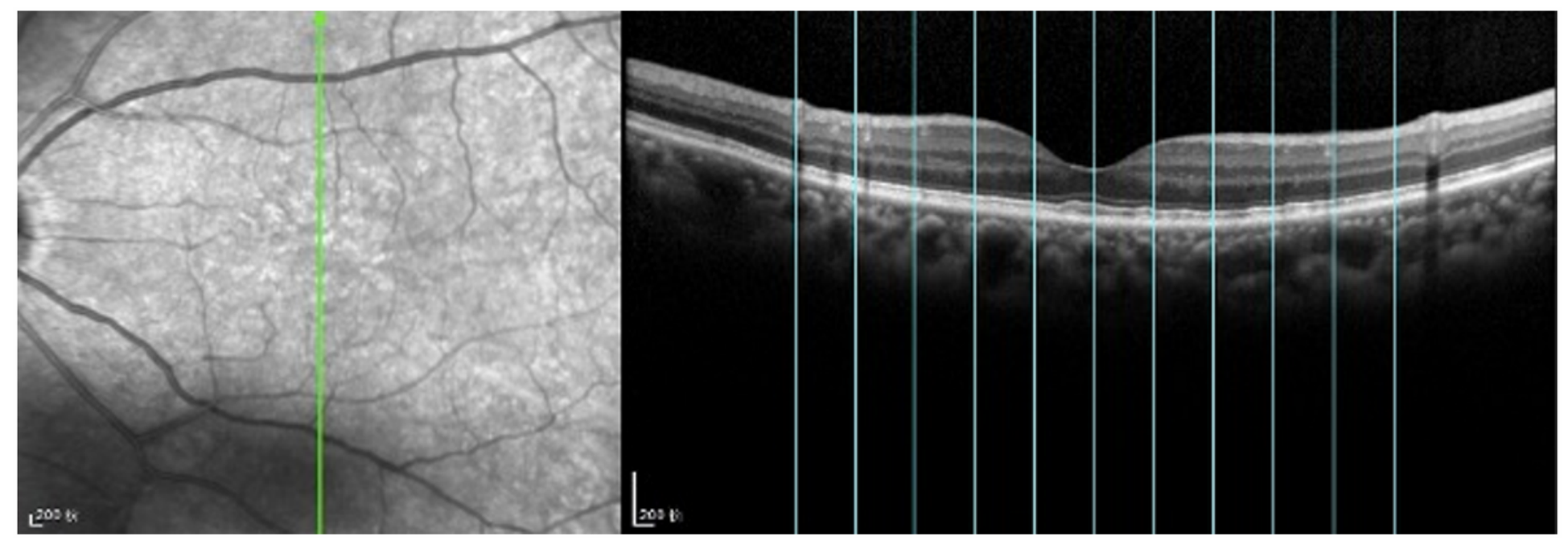

\section{Figure 1}

Scans of all samples using the German Heidelberg frequency domain optical coherence tomography. The green arrow is in the $90^{\circ}$ scan direction. The intersection of the blue line and the ellipsoid band is the measurement point.
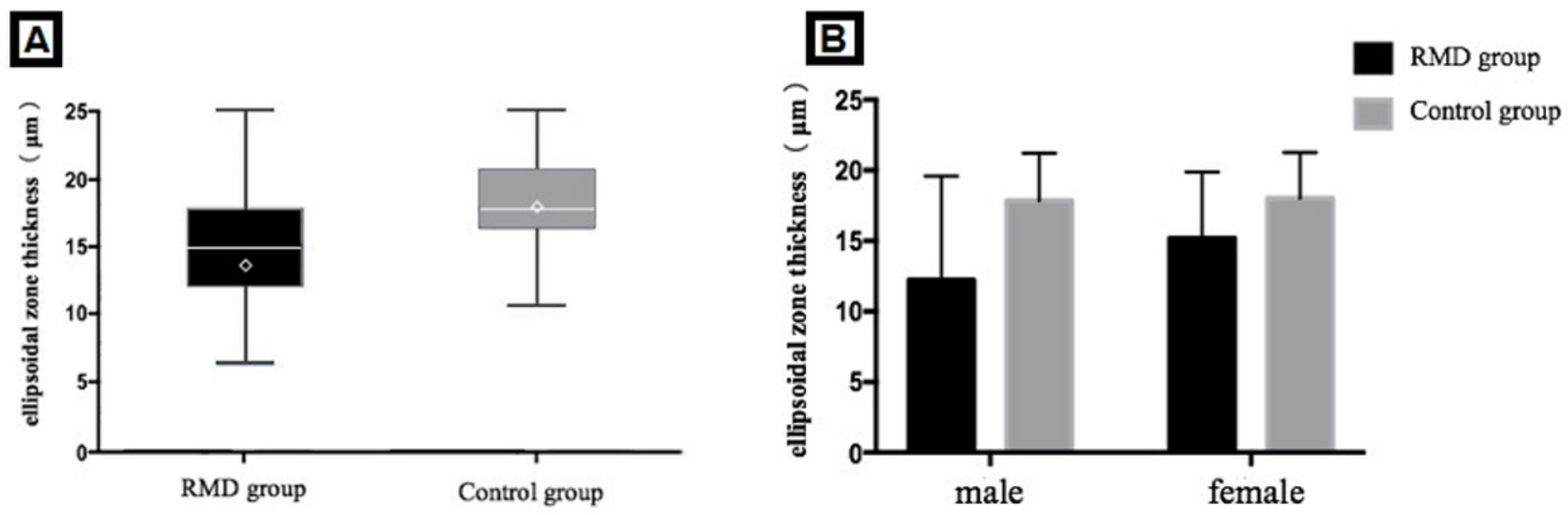

Figure 2 


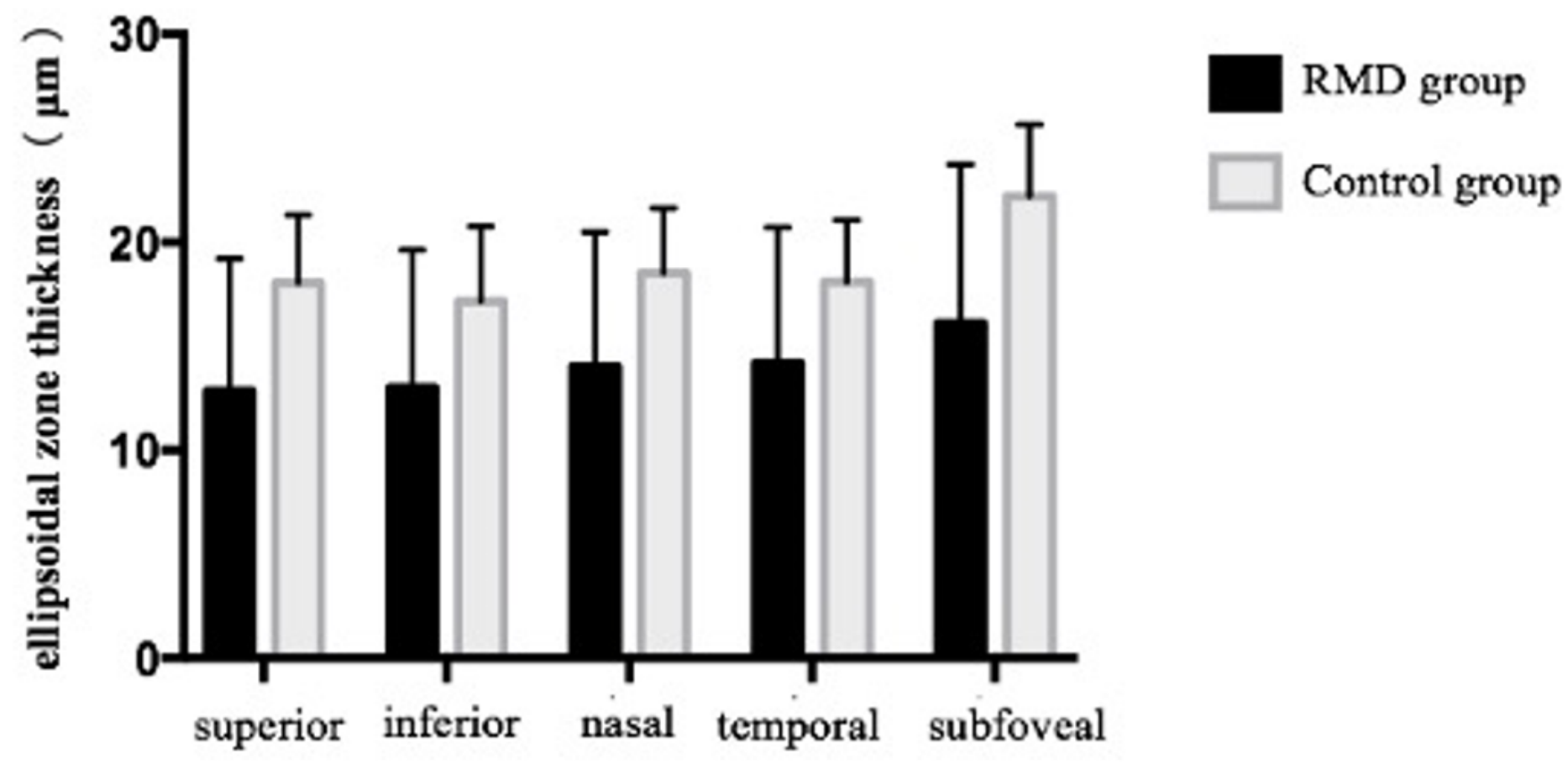

Figure 3

Comparison of ellipsoidal zone thickness in different quadrants between the Reticular Macular Disease (RMD) group and the control group. 